\title{
STRUCTURAL ASPECTS OF PHOTOCHROMISM OF THE INTERNALLY H-BONDED SCHIFF BASES. "A MINIMAL CHROMOPHORE"
}

\author{
A. Grabowska, K. Kownacki \\ Institute of Physical Chemistry, Polish Academy of Sciences \\ Kasprzaka 44/52, 01-224 Warsaw, Poland \\ AND E. KACZMAREK \\ Pharmaceutical Research Institute, Rydygiera 8, 01-793 Warsaw, Poland
}

Dedicated to Professor Zdzistaw Ruziewicz on the occasion of his 7olh birthday*

(Received July 4, 1995)

The final limiting stage of structural simplification of photochromic Schiff base was reached by the following statement: two molecules, salicylidene methylamine and its butyl analogue, show transient absorption bands (at room temperature) peaking at $\approx 22000 \mathrm{~cm}^{-1}$. These bands are characteristic of the phenomenon of photochromism. They are similar to the corresponding spectra detected earlier for several larger, symmetric systems belonging to the same family. Experimental characterization of both molecules was compared with the theoretical calculations: geometry optimization and transition energies, performed for enol, keto, and the photochromic form of salicylidene methylamine.

PACS numbers: $33.10 .-n, 33.50 .-\mathrm{j}$

\section{Introduction}

Photochromism of molecules belonging to the family of internally H-bonded Schiff bases was for many years studied almost exclusively on the example of salicylidene aniline (SA) and its simple derivatives; the majority of data concerned the solid state or low temperature studies [1].

The new impulse and a real progress have been recently noticed due to the introduction of the new compounds by the Japanese group of Maruyama and co-workers [2], and from other side - to the fact that time resolved techniques of vibrational spectroscopy have become available $[3,4]$.

*Submitted on invitation of the Institute of Physical and Theoretical Chemistry, Technical University of Wrocław, Wrocław, Poland. 
In a series of our recent papers [5-8] we studied a large family of Schiff bases, partly synthesised for the first time, partly kindly presented by Prof. Maruyama and Dr Inabe. The main aim was to compare structures possessing two equivalent proton transfer (PT) reaction sites with simpler systems that could be regarded as "halves" of their symmetric counterparts. The examples are shown in Fig. 1.

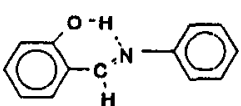

SA

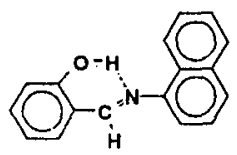

SN

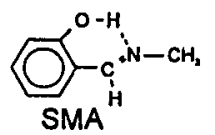

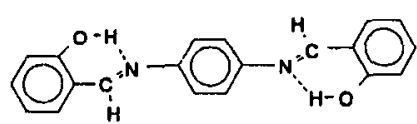

BSP
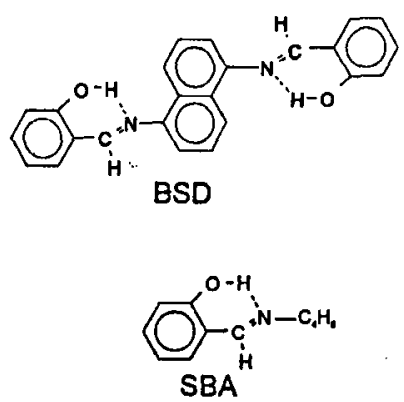

Fig. 1. SA: $N$-salicylidene aniline, BSP: $N, N^{\prime}$-bis(salicylidene)-p-phenylenediamine, SN: $N$-salicylidene-1-naphthylamine, BSD: bis(salicylidene)-1,5-diaminonaph thalene, SMA: salicylidene methylamine, SBA: salicylidene butylamine.

The main conclusion was [5-8] that the spectral manifestation of photochromism, e.g. the long living transient absorption, the time resolved resonance Raman response, as well as two fluorescences of two different transients, are surprisingly independent of the overall structure of the molecule. In contrast, the ground state absorption spectra "felt" very well the extent of conjugation of the absorbing molecules.

From these observations it was deduced that in symmetric Schiff bases only one proton is transferred in $S_{1}$ state, triggering the photochromic PT cycle of the molecule that involved a strongly localised photochromic transient (Fig. 2).

In the present work an attempt is made to synthesise the photochromic Schiff base as simple as possible, still revealing all manifestations of photochromism.

As will be shown below, simple structures, salicylidene methylamine(SMA), and its butyl analogue (SBA) were synthesised and compared with more complex molecules reported earlier.

The literature data on chemistry and spectroscopy of SMA are scarce. Besides the synthesis [9], only one paper [10], according to our best knowledge, reporting on the ground state enol $\rightleftharpoons$ keto equilibrium studied with absorption and Raman spectra as a function of temperature, was published. The conclusion was that in a protic, methanol solution both forms coexist. The zwitterionic form of 


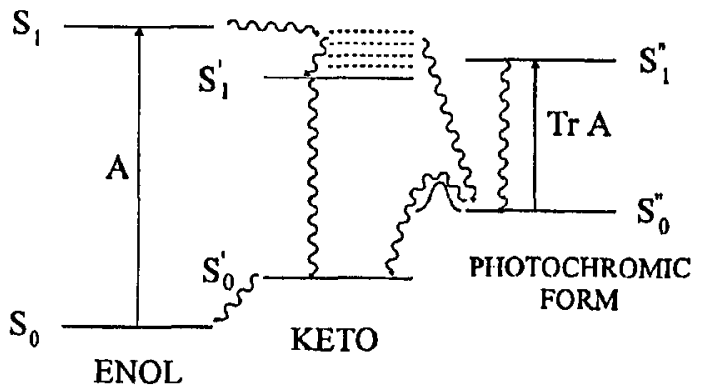

Fig. 2. Proton transfer photochromic cycle of SMA molecule.

keto tautomer was believed to represent the product of tautomerization, preferentially stabilised at low temperatures. The vibrational analysis of the corresponding Raman spectra was not able to assign unambiguously the zwitterionic species.

In summary, general opinion extracted from literature [11-13] concerning large systems of Fig. 1, like BSP, SA, etc. is the following: The tautomer, stable in the ground state, in neutral and polar nonprotic solvents, is an enol form; the tautomeric equilibrium can be shifted to a keto (zwitterionic ?) tautomer only in protic, alcohol solvents.

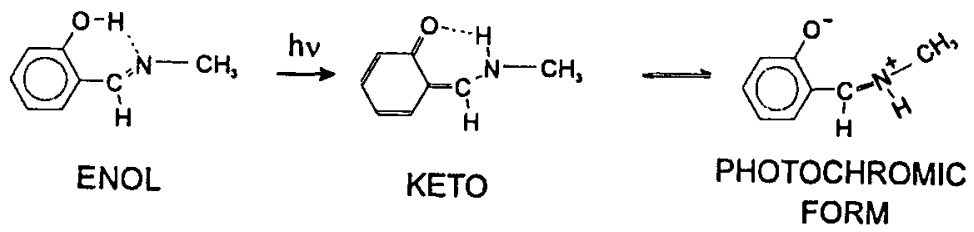

Fig. 3. Photochromism of SMA molecule.

In the present work we focused our attention on few questions only:

(1) How much can we simplify the structure of the Schiff base still revealing the phenomenon of photochromism (Fig. 3)? This question comes directly from our previous studies showing that in a large series of molecules belonging to this family, excitation is strongly localized.

(2) Which is the spectral characteristics of this "minimal photochromic" compound?

(3) Is it possible to observe its keto tautomer in the ground state in the gently interacting, e.g., nonprotic solvent?

The most general aim of the present work can be stressed as follows: The phenomenon of photochromism of Schiff bases suffers for the lack of proper assignment of the long living transient responsible for the absorption in the visible region. The simplification of the molecular framework still preserving the fundamental properties of photochromic systems would open the field to the most advanced modern quantum chemical methods of calculation of their electronic and vibrational structure. 


\section{Experimental technique and theoretical methods}

All instrumental tools used for the standard absorption and fluorescence spectra, as well as transient absorption, were the same as described elsewhere $[5,6]$. Solvents:

- acetonitrile - the following procedure was used in order to remove traces of water: $\mathrm{CII}_{3} \mathrm{CN}$ (Merck, Uvasol) was kept for 24 hours over $\mathrm{P}_{2} \mathrm{O}_{5}$, then after separation of the precipitate refluxed over $\mathrm{CaII}_{2}$ for 4 hours and finally distilled under argon. All subsequent manipulations were carried out in a drybox.

- benzene (POCh Gliwice) was used after distillation.

- cyclopentane (Fluka for UV spectroscopy) was used without further purification.

Theoretical calculations were composed of two parts: first, a ground state structure was optimized with application of the molecular mechanics method (MMX), and then $S_{0} \rightarrow S_{1}$ transitions of the enol, keto and zwitterionic photochromic forms were calculated with INDO/S method.

\section{Results}

\subsection{Synthesis}

First, an attempt was made do synthesise the simplest structure, from the three shown in Fig. 4, the compound 1, trying to follow an old paper by Ettling [14]. The following description makes clear, why this turned out to be impossible.

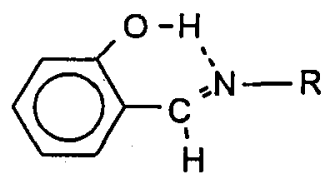

$$
\begin{aligned}
& R=H: 1 \\
& R=C_{3}: 2 \\
& R=C_{4} H_{8}: 3
\end{aligned}
$$

Fig. 4. The structure of salicylaldimine (2-iminomethylphenol) and of the derivatives investigated in this work.

The synthesis of salicylaldimine (2-iminomethylphenol) 1 was firstly attempted by C. Ettling in 1840 [14]. However, the reaction of salicylaldehyde with ammonia gave the compound of uncertain structure $\left(\mathrm{C}_{42} \mathrm{H}_{36} \mathrm{~N}_{4} \mathrm{O}_{6}\right)$, instead of the desired structure 1 . Till now only complexes of 1 with different metals are known and pure salicylaldimine was not isolated. Therefore we decided to synthesise $N$-methyl derivative of 1 (2) [9] as the simplest model compound containing o-iminomethylphenolic structure, as well as its $N$ - $n$-butyl analogue, 3 [15].

o-(Methyliminomethyl)phenol (salicylidene methylamine, SMA) [9]. Methylamine water solution $(33 \%, 15 \mathrm{~g})$ was carefully added to stirred salicylaldehyde $(12.2 \mathrm{~g}, 0.1 \mathrm{~mol})$ and the mixture was left overnight at room temperature. Then ether $(100 \mathrm{ml})$ was added to the mixture and the water layer was saturated with $\mathrm{NaCl}$. The ether layer was separated, dried over anhydrous $\mathrm{MgSO} 4$, evaporated 
and the residue distilled to afford yellow oil (b.p. $145^{\circ} \mathrm{C} / 20 \mathrm{~mm} \mathrm{IIg}$ ), yield $11 \mathrm{~g}$ $(81 \%)$.

o-(Bulyliminomethyl)phenol (salicylidene butylamine, $S B A$ ) [15]. The solution of salicylaldehyde $(12.2 \mathrm{~g}, 0.1 \mathrm{~mol})$ and $n$-butylamine $(7.3 \mathrm{~g}, 0.1 \mathrm{~mol})$ in benzene $(100 \mathrm{ml})$ was refluxed for 2 hrs using an apparatus for azeotropic distillation. Then the solvent was evaporated and the residue distilled to give yellow oil (b.p. $\left.84^{\circ} \mathrm{C} / 0.1 \mathrm{~mm} \mathrm{IIg}\right)$, yield $16.9 \mathrm{~g}(96 \%)$.

In our attempt to purify both synthesised compounds, SMA and SBA, it was stated that both decompose and recombine easily during, and after distillation. Both were stable enough to be studied photophysically.

\subsection{Spectra and photophysics of SMA and SBA}

Figures 5 and 6 show the summary of the results. The steady state absorption spectrum (Fig. 5), almost identical for SMA and SBA, is very well reproduced by the INDO/S calculations performed for the enol tautomer. Its geometry was obtained with application of MMX and is shown in Fig. 7. The satisfactory agreement of the calculated and observed transitions encrgics corresponding to the three maxima $\left(\nu_{1} \approx 32000 \mathrm{~cm}^{-1}, \nu_{2} \approx 39590 \mathrm{~cm}^{-1}, \nu_{3} \approx 46640 \mathrm{~cm}^{-1}\right)$ of absorption curve support the common opinion that the enol tautomer is the dominant ground state "version" of internally II-bonded Schiff base. Figure 5 shows, however, something else, the very weak absorption at about $25000 \mathrm{~cm}^{-1}$ and the "mirror imaging" fluorescence must be assigned as spectral fingerprints of the keto tautomer. Here we detect the characteristic long wave absorption band, nicely reproduced by the INDO/S calculation performed for the keto form shown in Fig. 3, and similar to the band reported in Ref. [10]. In our experiment the presence of keto tautomer is stated in a carefully dried, nonprotic solvent, $\mathrm{CII}_{3} \mathrm{CN}$. The excitation within this long wave $\left(\nu_{\max } \approx 25000 \mathrm{~cm}^{-1}\right)$ absorption band gives rise to the keto-tautomeric fluorescence. So, the product of the ground state tautomerization reaction of SMA may be observed in nonprotic solvent as well.

In contrast to other systems of Fig. 1, neither in SMA nor in SBA, the excited state proton transfer can be observed directly. The corresponding keto-tautomeric, largely Stokes shifted fluorescence is so weak in these molecules in tlie neutral solvents that it cannot be analysed quantitatively.

According to the experience from the earlier studied Schiff bases, the most efficient route of the excited PT reaction product is the population of the photochromic transient in its ground state. The generation route of this long living transient is presented in the introduction (Fig. 2).

Figure 6 shows the photochromic transient absorption spectra of SMA and SBA together with the standard $S_{0} \rightarrow S_{1}$ absorptions, as compared with other Schiff bases shown in Fig. 1.

First, the effect of photochromism in two "small" internally H-bonded Schiff bases is definitely stated, as transient absorption curves in Fig. 6 show. The stationary absorption spectra collected in Fig. 6 show the easily predictable and expected "consciousness" of the molecule of the extent of its $\pi$-electron system, while the photochromic absorption is remarkably non sensitive to the overall structure of absorbing molecule. This observation led us earlier to the conclusion that in pho- 

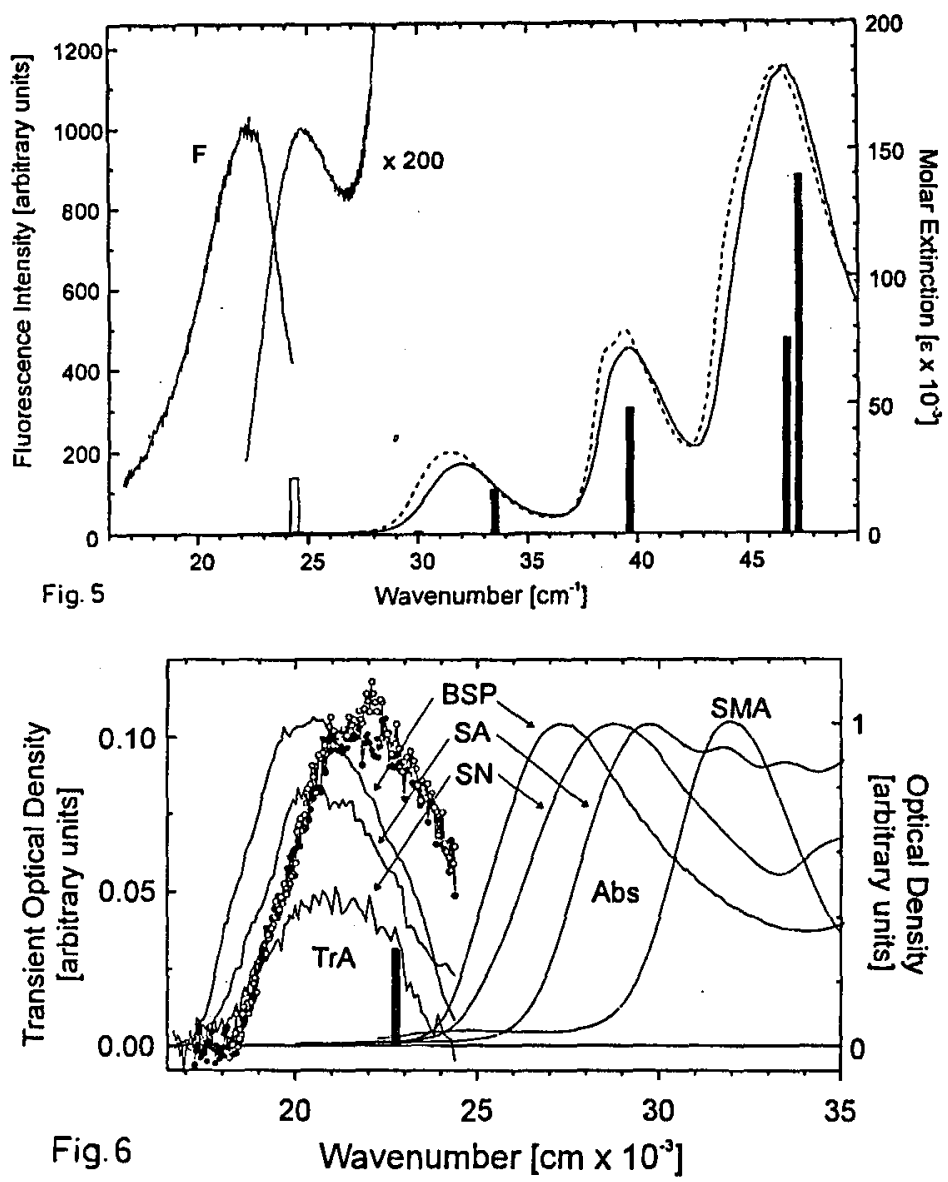

Fig. 5. Absorption and fluorescence spectra of SMA. Full line - acetonitrile solution, dashed line - cyclopentane. F - fluorescence spectrum. Full bars represent calculated transitions for enol form, empty bar that for keto tautomer.

Fig. 6. Abs - ground state absorption spectra of SMA, SA, SN and BSP in acetonitrile, TrA - transient absorption spectra, ०-०-॰-SMA in benzene, $\bullet-\bullet--S B A$ in benzene. Full bar - calculated transition energy for the photochromic form of SMA.

tochromic Schiff bases the electronic excitation and excited state intramolecular proton transfer reaction are strongly localised. The molecules of the present study fit well to this generalization, although their transient absorption spectra are definitely shifted by $1000-2000 \mathrm{~cm}^{-1}$ to the blue in respect to other systems. The bar in Fig. 6 marks the transition energy calculated for the zwitterionic form of the tautomerization product shown in Fig. 7. The examination of Fig. 6 shows another interesting behaviour: the spectral distance between the transient and the stationary absorption band is particularly large in the case of SMA and SBA. Interpretation of the above experimental findings will follow in the next chapter. 


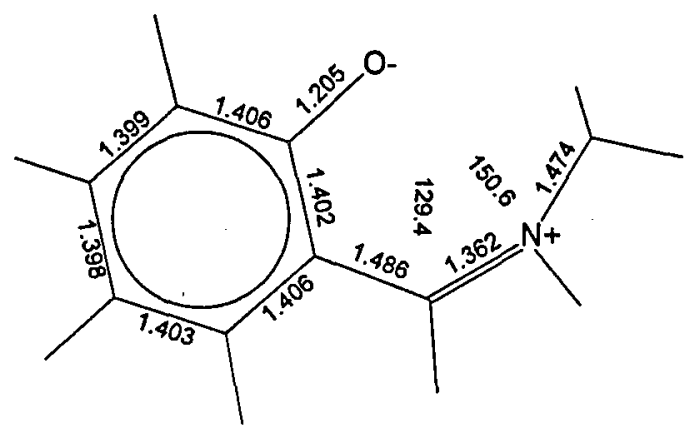

Fig. 7. Optimized by molecular mechanics (MMX) geometry of the photochromic form of SMA molecule.

\section{Discussion}

In the present work we believe to have reached the final stage of simplification of the photochromic Schiff base. As it was shown in the introduction, the molecule 1 of Fig. 4 is unstable and cannot be separated as a monomer. So, we have in hand the molecule SMA, still simple enough to be the attractive object for the advanced theoretical calculations of the electronic and vibrational structure. A common chromophore of all photochromic Schiff bases postulated in earlier studies $[5-8]$ is most probably fairly well represented by SMA molecule. The remarkable localization of the electronic excitation and the proton transfer reactivity leading to the photochromic transient, is now a matter of experimental evidence.

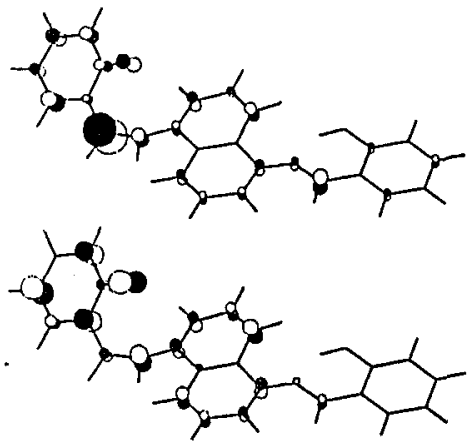

BSD
LUMO<smiles>CC1C(C)C(C)C(OC(C)(C)C(C)(C)C)C(C)C1C</smiles>

HOMO<smiles>CC(C)=C(C)C(C)=C(C)C(C)=C(C)C</smiles>

SMA

Fig. 8. HOMO and LUMO orbitals involved in $S_{0} \rightarrow S_{1}$ transition of photochromic forms of BSD and SMA obtained from INDO/S calculations. Visualization prepared by MOPLOT program [16]. 
Moreover, in SMA and SBA molecules, it was shown that the "unusual" keto tautomer can be stabilized also in a dry, nonprotic polar solvent with exclusion of the intermolecular II-bonds, while in other systems, like BSP or SA, such ground state tautomerization was observed only in alcoholic solvents [11, 12]. Figures 5 and 6 show the accuracy of calculations of transition energies performed for the keto and zwitterionic forms, respectively. The direct comparison of theory and experiment leaves no doubt that two forms of PT reaction product are easily. distinguishable.

The last interesting point is illustrated by Fig. 8, comparing the HOMOLUMO orbitals of the zwitterionic transients of BSD and SMA. BSD molecule is chosen as an example of a more extended system which was demonstrated to generate a localized photochromic transient. In Fig. 8 it is seen that this localization is not quite exact, and in fact, the "excitation is more extended" than in SMA molecule. This may be the reason; why photochromic transient absorption bands of SMA and SBA are shifted to the blue in respect to larger systems, as it is illustrated in Fig. 6.

The study of time resolved vibrational spectra of SMA will be the matter of our next paper.

\section{Acknowledgment}

This work was partly supported (synthesis, purification of SMA and SBA) by the grant 2P303 11104 of Committee for Scientific Research.

\section{References}

[1] E. Hadjoudis, in: Photochromism. Molecules and Systems, Eds. H. Dürr, H. Bouas-Laurent, in series Studies in Organic Chemistry, Vol. 40, Elsevier, Amsterdam 1990.

[2] N. Hoshino, T. Inabe, T. Mitani, Y. Maruyama, Bull. Chem. Soc. Jpn. 61, 4207 (1988).

[3] T. Yuzawa, II. Takahashi, H. Hamaguchi, Chem. Phys. Lett. 202, 221 (1993).

[4] W. Turbeville, P.K. Dutta, J. Phys. Chem. 94, 4060 (1990).

[5] K. Kownacki, Ł. Kaczmarek, A. Grabowska, Chem. Phys. Lett. 210, 373 (1993).

[6] K. Kownacki, A. Mordziński, R. Wilbrandt, A. Grabowska, Chem. Phys. Lett. 227, 270 (1994).

[7] A. Grabowska, K. Kownacki, Ł. Kaczmarek, J. Lumin. 60\&61, 886 (1994).

[8] A. Mordziński, K. Kownacki, R. Wilbrandt, C. Rios, J. Inf. Rec. Mats. 21, 601 (1994).

[9] N.H. Cromwell, II. Hoeksma, J. Am. Chem. Soc. 67, 1658 (1945).

[10] H. Lee, T. Kitagawa, Bull. Chem. Soc. Jpn. 59, 2897 (1986).

[11] M. Melzig, Ph.D. Thesis, Technische Universität, München 1981.

[12] R. Potashnik, M. Ottolenghi, J. Chem. Phys. 51, 3671 (1969).

[13] T. Rosenfeld, M. Ottolenghi, A.Y. Meyer, Mol. Photochcm. 5, 39 (1973).

[14] C. Ettling, Ann. Chem. Pharm. 35, 241 (1840).

[15] L.H. Goodson, II. Christopher, J. Am. Chem. Soc. 71, 1117 (1949).

[16] MOPLOT Program, T. Bally, Fribourg University. 